\title{
PERMUTATIONS WITH EQUAL ORDERS
}

\author{
HUSEYIN ACAN, CHARLES BURNETTE ${ }^{1}$, SEAN EBERHARD, ERIC SCHMUTZ, \\ AND JAMES THOMAS ${ }^{2}$
}

\begin{abstract}
Let $\mathbf{P}\left(\right.$ ord $\pi=$ ord $\left.\pi^{\prime}\right)$ be the probability that two independent, uniformly random permutations of $[n]$ have the same order. Answering a question of Thibault Godin, we prove that $\mathbf{P}\left(\operatorname{ord} \pi=\operatorname{ord} \pi^{\prime}\right)=$ $n^{-2+o(1)}$ and that $\mathbf{P}\left(\right.$ ord $\pi=$ ord $\left.\pi^{\prime}\right) \geqslant \frac{1}{2} n^{-2} \lg ^{*} n$ for infinitely many $n$. (Here $\lg ^{*} n$ is the height of the tallest tower of twos that is less than or equal to $n$.)
\end{abstract}

\section{INTRODUCTION}

1.1. The problem. Let $\pi$ be a random permutation of $[n]$. Write ord $\pi$ for the order of a permutation $\pi$, i.e., the least common multiple of its cycle lengths. The distribution of ord $\pi$ is an object of basic interest in probabilistic group theory. For example, a beautiful theorem of Erdős and Turán [ET67] asserts that $\log$ ord $\pi$ is asymptotically normal with mean $\log ^{2} n / 2$ and variance $\log ^{3} n / 3$. Many more features of the distribution of ord $\pi$ are visible through the lens of the theory of logarithmic combinatorial structures: see for example the book of Arratia, Barbour, and Tavaré [ABT03]. For example, the largest cycles of $\pi$, which determine the magnitude of ord $\pi$ and its divisibility by large primes, follow a PoissonDirichlet law.

A more subtle feature of the distribution of ord $\pi$ is its collision entropy. Recall that the collision entropy or Rényi 2-entropy of a random variable $X$ is defined by

$$
H_{2}(X)=-\log \mathbf{P}\left(X=X^{\prime}\right)
$$

where $X^{\prime}$ is an independent copy of $X$. In other words, for $X=\operatorname{ord} \pi$, the problem is to estimate

$$
e^{-H_{2}(\operatorname{ord} \pi)}=\mathbf{P}\left(\operatorname{ord} \pi=\operatorname{ord} \pi^{\prime}\right) .
$$

This problem was highlighted recently by Godin God17] in connection with automaton groups. We are grateful to Sergey Dovgal for bringing this problem to our attention.

\footnotetext{
${ }^{1}$ Some of this work was done while Charles Burnette was a postdoctoral visitor at Saint Louis University and the Institute of Statistical Science, Academia Sinica.

${ }^{2}$ Portions of this work appear in James Thomas' Ph.D. thesis at Drexel University.
} 
Let type $\pi$ denote the cycle type of $\pi$, i.e., the multi-set of its cycle lengths. Since permutations with the same type have the same order, it is clear that

$$
\mathbf{P}\left(\text { ord } \pi=\operatorname{ord} \pi^{\prime}\right) \geqslant \mathbf{P}\left(\text { type } \pi=\text { type } \pi^{\prime}\right) \text {. }
$$

Using methods of analytic combinatorics, it was proved by Flajolet, Fusy, Gourdon, Panario, and Pouyanne $\left[\mathrm{FFG}^{+} 06\right.$, Proposition 4] that

$$
\mathbf{P}\left(\text { type } \pi=\text { type } \pi^{\prime}\right)=\frac{c_{0}}{n^{2}}+O\left(\frac{1}{n^{3}}\right), \quad c_{0}=\prod_{k \geqslant 1} I\left(\frac{1}{k^{2}}\right) \approx 4.26,
$$

where $I(z)=\sum_{n \geqslant 0} z^{n} / n !^{2}$. Based on this lower bound and computations, Godin conjectured that

$$
\lim _{n \rightarrow \infty} n^{2} \mathbf{P}\left(\text { ord } \pi=\text { ord } \pi^{\prime}\right)=K
$$

for some constant $K$ with $c_{0} \leqslant K \leqslant 12$ (see God17, Conjecture 15]).

It follows from the Erdős-Turán limit law for $\log$ ord $\pi$ that $\mathbf{P}($ ord $\pi=$ ord $\left.\pi^{\prime}\right)=o(1)$, but establishing any explicit rate of decay is already nontrivial. A crude bound was established in an earlier version of this paper and in the fifth author's thesis [Tho20. Briefly, using estimates for the probability that ord $\pi$ is coprime to a given integer, one can prove that with high probability there is a prime in the interval $[\log n, 2 \log n]$ that divides exactly one of ord $\pi$ and ord $\pi^{\prime}$. This argument leads to a bound of the form $O(\log \log n / \log n)$, but does not come close to Godin's conjecture.

We can contrast the effort involved in estimating the collision entropies of type $\pi$ and ord $\pi$ even further. Suppose $k=o(n)$ and let $\lambda=\left\langle 1^{\lambda_{1}}, 2^{\lambda_{2}}, \ldots\right\rangle$ be a partition of $k$. Then

$\mathbf{P}\left(\pi\right.$ and $\pi^{\prime}$ have type $\left.\langle\lambda, n-k\rangle\right)=\frac{1}{(n-k)^{2}} \prod_{j \geqslant 1} \frac{1}{j^{2 \lambda_{j}}\left(\lambda_{j} !\right)^{2}} \approx \frac{1}{n^{2}} \prod_{j \geqslant 1} \frac{1}{j^{2 \lambda_{j}}\left(\lambda_{j} !\right)^{2}}$.

Heuristically summing the rightmost approximation over all such $k$ and $\lambda$ yields a substantial partial sum of

$$
\frac{1}{n^{2}} \sum_{k \geqslant 0} \sum_{\lambda \vdash k} \prod_{j \geqslant 1} \frac{1}{j^{2 \lambda_{j}}\left(\lambda_{j} !\right)^{2}}=\frac{c_{0}}{n^{2}}
$$

where $\lambda \vdash k$ has the usual meaning that $\lambda$ is a partition of $k$ (i.e. a multiset of positive integers whose sum is $k$ ). This together with the analysis of $\left[\mathrm{FFG}^{+} 06\right]$ shows that the main contribution to $n^{2} \mathbf{P}\left(\right.$ type $\pi=$ type $\left.\pi^{\prime}\right)$ comes from pairs of permutations having a cycle of length $n-o(n)$. Motivated by this, one may ask whether at least $n^{2} \mathbf{P}\left(\operatorname{ord} \pi=\operatorname{ord} \pi^{\prime} \wedge E\right)$ is bounded, where $E$ is the event that $\pi$ and $\pi^{\prime}$ each have a cycle of length at least $n-k(n)$, where $k(n)$ is some slowly growing function. This, however, is not the case.

In this paper we prove two main results. First, we refute (1) by showing that

$$
\limsup _{n \rightarrow \infty} n^{2} \mathbf{P}\left(\operatorname{ord} \pi=\text { ord } \pi^{\prime}\right)=\infty
$$


Quantitatively, we show that there is a sequence $n_{i} \rightarrow \infty$ such that if $\pi, \pi^{\prime}$ are drawn independently from $S_{n_{i}}$ then

$$
\mathbf{P}\left(\operatorname{ord} \pi=\operatorname{ord} \pi^{\prime}\right) \geqslant \mathbf{P}\left(\operatorname{ord} \pi=\operatorname{ord} \pi=n_{i}-o\left(n_{i}\right)\right) \geqslant \frac{1}{2} n_{i}^{-2} \lg ^{*} n_{i},
$$

where $\lg ^{*} n$ is the height of the tallest tower of twos that does not exceed $n$. This precludes any heuristic similar to (2) and (3) from succeeding here. On the other hand we show that (10) is nearly true, in the sense that

$$
\mathbf{P}\left(\operatorname{ord} \pi=\operatorname{ord} \pi^{\prime}\right) \leqslant n^{-2+o(1)} .
$$

It would be interesting to estimate $\mathbf{P}\left(\operatorname{ord} \pi=\operatorname{ord} \pi^{\prime}\right)$ more precisely, but this appears to be a complicated question tied to arithmetic considerations about $n$.

For a broader perspective, readers may be interested in the survey of Niemeyer, Praeger, and Seress on the applications of probabilistic and enumerative techniques to the analysis of group-theoretic algorithms [NPS13.

1.2. Analytic Combinatorics. Analytic combinatorics relates the analytic behaviour of a generating function to the asymptotic behaviour of its coefficients. While the problem of estimating $\mathbf{P}$ (type $\pi=$ type $\left.\pi^{\prime}\right)$ is wellsuited to the methods of analytic combinatorics, the same does not seem to be true of $\mathbf{P}\left(\operatorname{ord} \pi=\right.$ ord $\left.\pi^{\prime}\right)$. We offer some brief comments about why this may be.

Elementary combinatorial techniques are sufficient for enumerating the ordered pairs of conjugate permutations. As a result, the numbers $\mathbf{P}$ (type $\pi=$ type $\pi^{\prime}$ ) are expressible as the coefficients of a well-behaved infinite product generating function closely related to the cycle index of the symmetric group (as explained in $\left[\mathrm{FFG}^{+} 06\right.$, Section 4.2$]$.

In contrast consider $\mathbf{P}\left(\operatorname{ord} \pi=\right.$ ord $\left.\pi^{\prime}\right)$. For any fixed positive integer $m$, the exponential formula yields

$$
F_{m}(x)=\sum_{n} \mathbf{P}(\operatorname{ord} \pi \text { divides } m) x^{n}=\exp \left(\sum_{d \mid m} \frac{x^{d}}{d}\right) .
$$

An application of Möbius inversion to (5) thus yields

$$
G_{m}(x)=\sum_{n} \mathbf{P}(\operatorname{ord} \pi=m) x^{n}=\sum_{d \mid m} \mu\left(\frac{m}{d}\right) F_{d}(x) .
$$

Using Möbius and Lagrange inverson, and the saddle-point method, Wilf [Wil86] used (6) to derive an asymptotic formula for $\mathbf{P}($ ord $\pi=m)$ for fixed $m$, but as $m$ grows Wilf's formula becomes more complicated and the asymptotics are less well-understood. In the special case of $m=n$ there is a theorem of Warlimont War78] that

$$
\mathbf{P}(\operatorname{ord} \pi=n)=1 / n+O\left(1 / n^{2}\right),
$$

and this estimate has been extended by Niemeyer and Praeger [NP07] to various other values of $m$. A general understanding of $\mathbf{P}(\operatorname{ord} \pi=m)$ is 
lacking, and indeed complicated for arithmetic reasons. As such, one cannot simply plug these asymptotic estimates into the $\operatorname{sum} \sum_{m} \mathbf{P}(\operatorname{ord} \pi=m)^{2}$ to answer Godin's question.

There is a rich literature about methods for extracting the coefficients of multivariate generating functions [PW13, Rai11. Certainly we may define a bivariate generating function $H(x, y)=\sum_{m} G_{m}(x) G_{m}(y)$, and

$$
\mathbf{P}\left(\operatorname{ord} \pi=\operatorname{ord} \pi^{\prime}\right)=\left[\left[x^{n} y^{n}\right]\right] H(x, y) .
$$

In some formal sense this is an answer, but we do not see any way to extract an asymptotic formula from (17).

Analytic combinatorics, by itself, is likely inadequate for attaining a thorough asymptotic analysis of the sequence $\mathbf{P}($ ord $\pi=m)$ because the order of a permutation depends on arithmetic data not easily extracted from the classical generating functions associated with permutations. Any hope for a purely symbolic calculus that can handle the sequence $\mathbf{P}\left(\operatorname{ord} \pi=\operatorname{ord} \pi^{\prime}\right)$ might hinge on techniques that are more in the realm of analytic number theory, such as a Mellin transform or a Dirichlet series generating function.

Another notable obstruction to a generating-function-based approach is the apparently erratic dependence of $\mathbf{P}\left(\operatorname{ord} \pi=\operatorname{ord} \pi^{\prime}\right)$ on $n$, which may be observed numerically. If the sequence $\mathbf{P}\left(\operatorname{ord} \pi=\operatorname{ord} \pi^{\prime}\right)$ were realized as the coefficients of a generating function, the behaviour of that function near its singularity would have to be similarly complicated.

1.3. The anatomy of integers. In sharp relief from the beautiful formalism of analytic combinatorics, our proof of (4) is dirty and hands-on, and more closely connected with the "anatomy of integers": see Granville Gra for an explanation of this term, and Ford [For] or the book of Hall and Tenenbaum [HT88, for a sense of the scope of the theory. We have mentioned already that $\log \operatorname{ord} \pi$ is asymptotically normal with mean $\log ^{2} n / 2$ and variance $\log ^{3} n / 3$, and that the largest cycles of $\pi$ are distributed asymptotically according to the Poisson-Dirichlet law. By further analyzing the distribution of the cycles of $\pi$ we show that apart from an exceptional event of probability $n^{-1+o(1)}$, including for instance the event that $\pi$ is an $n$-cycle or an $(n-1)$-cycle, the integer $m=$ ord $\pi$ will have many large prime divisors, so many in fact that the collision probability $\mathbf{P}\left(\right.$ ord $\left.\pi^{\prime}=m\right)$ is negligible. It follows that the probability that ord $\pi=\operatorname{ord} \pi^{\prime}$ is dominated by the event that $\pi$ and $\pi^{\prime}$ are both exceptional.

\section{Disproof of Godin's Conjecture}

The results in this section are based on the third author's mathoverflow post [Thi16]. Define Tow $(h)$ to be a tower of twos of height $h$, i.e., Tow $(0)=$ 1 , and for $h>0$, Tow $(h)=2^{\operatorname{Tow}(h-1)}$. Also define $\lg ^{*} n=\max \{h: \operatorname{Tow}(h) \leqslant$ $n\}$. 
Theorem 1. For infinitely many positive integers $n$,

$$
\mathbf{P}\left(\operatorname{ord} \pi=\operatorname{ord} \pi^{\prime}\right) \geqslant \frac{\lg ^{*} n}{2 n^{2}} .
$$

Proof. For a positive integer $n$, let $K_{n}=\{k: 1 \leqslant k<n / 2$ and $k$ ! divides $n-$ $k$ \}. If $\pi$ has a cycle of length $n-k$, with $k \in K_{n}$, then all other cycles have length at most $k$. Since the lengths of these other cycles are at most $k$, they all divide $k$ !, which in turn divides $n-k$ (by the definition of $K_{n}$ ). Therefore ord $\pi=n-k$. The probability that $\pi$ has a cycle of length $n-k$ is exactly $1 /(n-k)$. Since $n-k>\frac{n}{2}$, these events are disjoint, since there cannot be more than one cycle of length greater than $\frac{n}{2}$. We therefore have

$$
\mathbf{P}\left(\operatorname{ord} \pi=\operatorname{ord} \pi^{\prime}\right) \geq \sum_{k \in K_{n}} \frac{1}{(n-k)^{2}} \geq \frac{\left|K_{n}\right|}{n^{2}} .
$$

Now consider the subsequence $\left(n_{i}\right)_{i \geqslant 1}$ defined by $n_{1}=3$ and $n_{i+1}=n_{i}+n_{i}$ ! for $i \geq 1$. We will prove that the sets $K_{n_{i}}$ are nested and that $\left|K_{n_{i}}\right|=i$ for all $i$. From the definition of $K_{n}$, it is easy to check that

- $K_{n_{1}}=\{1\}$;

- $n_{i} \notin K_{n_{i}}$;

- $k \in K_{n_{i}} \Longrightarrow k \in K_{n_{i+1}}$, since if $k ! \mid n_{i}-k$ and $k \leqslant n_{i}$ then also $k ! \mid n_{i+1}-k$;

- $n_{i} \in K_{n_{i+1}}$, for the same reason.

Also note that

- $k \notin K_{n_{i+1}}$ for $k>n_{i}$ since $k$ ! is too big to divide $n_{i+1}-k$;

- if $k<n_{i}$ and $k \in K_{n_{i+1}}$, then we already have $k \in K_{n_{i}}$, since $k<n_{i} \Longrightarrow k ! \mid n_{i}$ !, which in turn implies $k ! \mid n_{i}-k$.

We therefore have $K_{n_{i+1}}=K_{n_{i}} \cup\left\{n_{i}\right\}$ and $n_{i} \notin K_{n_{i}}$, so inductively

$$
K_{n_{i}}=\left\{1, n_{1}, n_{2}, \ldots, n_{i-1}\right\} .
$$

This proves that $\left|K_{n_{i}}\right|=i$ for all $i$. Since $\left|K_{n_{i}}\right| \rightarrow \infty$ as $i \rightarrow \infty$, it is now clear that $\lim \sup n^{2} \mathbf{P}\left(\operatorname{ord} \pi=\operatorname{ord} \pi^{\prime}\right)=\infty$.

To finish proving Theorem 1, we need to find a lower bound for $i$ that is expressed in terms of $n_{i}$. Since $2^{n^{2}} \geq(n+1) ! \geq n !+n$ for any positive integer $n$, we have

$$
\begin{aligned}
\lg ^{*}\left(n_{i+1}\right)=\lg *\left(n_{i} !+n_{i}\right) & \leq \lg ^{*}\left(2^{n_{i}^{2}}\right) \\
& =1+\lg ^{*}\left(n_{i}^{2}\right) \\
& \leq 1+\lg ^{*}\left(2^{n_{i}}\right)=2+\lg ^{*}\left(n_{i}\right) .
\end{aligned}
$$

It follows from induction on $i$ that $\lg ^{*}\left(n_{i}\right) \leq 2 i$ or equivalently, $i \geq \lg *\left(n_{i}\right) / 2$. Hence

$$
\frac{\left|K_{n_{i}}\right|}{n_{i}^{2}}=\frac{i}{n_{i}^{2}} \geq \frac{\lg ^{*}\left(n_{i}\right)}{2 n_{i}^{2}}
$$




\section{Main Proposition and Proof Sketch}

Throughout let $\pi$ be a random permutation of $[n]$. Our main result is the following.

Theorem 3.1. There is a set $\mathcal{M}$ with the following properties.

(1) If $m \notin \mathcal{M}$ then $\mathbf{P}($ ord $\pi=m)=O\left(n^{-100}\right)$.

(2) $\mathbf{P}($ ord $\pi \in \mathcal{M}) \leqslant n^{-1+o(1)}$.

Although the proof of Theorem 3 is postponed, we can immediately deduce a non-trivial upper bound for the probability that two random permutations have the same order.

Corollary 3.2. $\mathbf{P}\left(\right.$ ord $\pi=$ ord $\left.\pi^{\prime}\right) \leqslant n^{-2+o(1)}$.

Proof. By considering whether the collision occurs in $\mathcal{M}$ or $\mathcal{M}^{c}$ we have

$$
\begin{aligned}
\mathbf{P}\left(\operatorname{ord} \pi=\operatorname{ord} \pi^{\prime}\right) & \leqslant \mathbf{P}(\operatorname{ord} \pi \in \mathcal{M})^{2}+\sum_{m \notin \mathcal{M}} \mathbf{P}(\operatorname{ord} \pi=m)^{2} \\
& \leqslant \mathbf{P}(\operatorname{ord} \pi \in \mathcal{M})^{2}+\max _{m \notin \mathcal{M}} \mathbf{P}(\operatorname{ord} \pi=m) .
\end{aligned}
$$

The first term is bounded by $n^{-2+o(1)}$ and the second term is bounded by $O\left(n^{-100}\right)$.

For the proof of Theorem 3, we construct a specific example of such a set $\mathcal{M}$. For the remainder of this paper, let $\delta=\delta(n)=1 / \log \log \log n$, and let $\eta=e^{-10 / \delta}=\frac{1}{(\log \log n)^{10}}$, though the specific choice is largely irrelevant: all we require is that $\delta$ and $\eta$ decay sufficiently slowly, with $\delta$ decaying much more slowly than $\eta$. Let $\mathcal{M}$ be the set of all positive integers $m$ having at most $\delta \log n$ distinct prime divisors $p>n^{\eta}$.

Let us now informally sketch the proof of Theorem 3 (some readers may prefer to skip ahead to the next section for the rigorous proofs). It suffices to consider the case where $\pi$ has $k \geqslant 2 \delta \log n$ cycles, because all except $n^{-1+o(1)}$ permutations have this property (recall $\delta=o(1)$ ). These $k$ cycles will be drawn at random from $\{1, \ldots, n\}$ according to a harmonic weighting (conditional on their sum being $n$ ). Using Mertens' third theorem to bound the harmonic weight of the set of $n^{\eta}$-smooth numbers, we expect at least half of our $2 \delta \log n$ cycles to fail to be $n^{\eta}$-smooth. Therefore we expect ord $\pi$ to be divisible by some $\delta \log n$ primes $p>n^{\eta}$, proving part (2) of Theorem 3 , The proof of part (1) is easier, and follows from a simple union bound over all the ways that the cycles of $\pi$ might be divisible by the primes dividing $m$.

\section{Proof of Theorem 3, Part (2)}

Write $Z=Z(\pi)$ for the number of cycles in a random $\pi \in S_{n}$. It is well known that $Z-1$ is approximately Poisson with parameter $\log n$. (See, for example, the final section of Dev88 for tail bounds.) The following lemma's quantitative formulation is particularly convenient for us. 
Lemma 2. Let $n, k \geqslant 1$, and let $\pi \in S_{n}$ be random. Then

$$
\mathbf{P}(Z(\pi)=k) \leqslant \frac{1}{n} \frac{h_{n}^{k-1}}{(k-1) !},
$$

where $h_{n}=\sum_{j=1}^{n} 1 / j$.

Proof. Write $p_{n, k}$ for $\mathbf{P}(Z(\pi)=k)$. From Cauchy's formula for the number of permutations in a conjugacy class, we have

$$
p_{n, k}=\sum \frac{1}{c_{1} ! \cdots c_{n} ! 1^{c_{1}} \cdots n^{c_{n}}},
$$

where the sum ranges over all $c_{1}, \ldots, c_{n} \geqslant 0$ such that $\sum_{i=1}^{n} c_{i}=k$ and $\sum_{i=1}^{n} i c_{i}=n$. We can "smooth this out" by using

$$
p_{n, k}=\frac{1}{n} \sum_{j=1}^{n} p_{n-j, k-1}
$$

which follows from conditioning on the length of one of the cycles of $\pi$. Thus we have

$$
\begin{aligned}
p_{n, k} & =\frac{1}{n} \sum_{j=1}^{n} \sum_{\substack{\sum c_{i}=k-1 \\
\sum i c_{i}=n-j}} \frac{1}{c_{1} ! \cdots c_{n} ! 1^{c_{1}} \cdots n^{c_{n}}} \\
& \leqslant \frac{1}{n} \sum_{\sum c_{i}=k-1} \frac{1}{c_{1} ! \cdots c_{n} ! 1^{c_{1}} \cdots n^{c_{n}}} \\
& =\frac{1}{n} \frac{h_{n}^{k-1}}{(k-1) !} .
\end{aligned}
$$

The last line is an application of the multinomial theorem.

Using Stirling's formula, and monotonicity of the bound $\frac{1}{n} \frac{h_{n}^{k-1}}{(k-1) !}$ as a function of $k$, we can prove the following corollary.

Corollary 3. The probability that $\pi$ has $o(\log n)$ cycles is $n^{-1+o(1)}$, and the probability that $\pi$ has more than $10 \log n$ cycles is $O\left(n^{-14}\right)$.

Proof. Let $\xi=\frac{h_{n}}{\omega}$, where $\omega=\omega(n) \rightarrow \infty$. By calculating the ratios of successive terms, one can verify that the bound $\frac{1}{n} \frac{h_{n}^{k-1}}{(k-1) !}$ is increasing as a function of $k$ when $k \leqslant \xi+1$. Thus

$$
\mathbf{P}(Z \leqslant \xi+1) \leqslant(\xi+1) \frac{1}{n} \frac{h_{n}^{\xi}}{(\xi+1) !} \leqslant \frac{1}{n}(e \omega)^{\xi}=n^{-1+o(1)} .
$$

Similarly, when $k>10 h_{n}$, the bound is decreasing as a function of $k$. In this range, a crude version of Stirling's formula yields

$$
\frac{h_{n}^{k-1}}{k !} \leqslant\left(\frac{e h_{n}}{k}\right)^{k} \leqslant\left(\frac{e}{10}\right)^{k}
$$


Therefore

$$
\mathbf{P}(Z \geqslant 10 \log n) \leqslant \frac{1}{n} \sum_{k \geqslant 10 h_{n}}\left(\frac{e}{10}\right)^{k}=O\left(n^{10 \log (e / 10)-1}\right) .
$$

We use only Corollary 3 in the proof, but a similar argument establishes that, for fixed positive $\epsilon$, the probability that $\pi$ has more than $(1+\epsilon) \log n$ cycles is bounded by $n^{-f(\epsilon)+o(1)}$, where $f(\epsilon)=(1+\epsilon) \log (1+\epsilon)-\epsilon$.

Lemma 4. Let $A_{1}, \ldots, A_{Z}$ be the cycle lengths of $\pi$ in a random order. Then for any $k \geqslant 0$ and any $k$-tuple $\left(a_{1}, \ldots, a_{k}\right)$ of positive integers such that $a_{1}+\cdots+a_{k}=n$ we have

$$
\mathbf{P}\left(Z=k, A_{1}=a_{1}, \ldots, A_{k}=a_{k}\right)=\frac{1}{k !} \frac{1}{a_{1} \ldots a_{k}} .
$$

Proof. Let the multiplicities among $a_{1}, \ldots, a_{k}$ be $m_{1}, \ldots, m_{s}$ (so that $\sum_{i} m_{i}=$ $k$ ). Then by Cauchy's formula the probability that this cycle type arises is

$$
\frac{1}{m_{1} ! \cdots m_{s} ! a_{1} \cdots a_{k}} \text {. }
$$

When these cycles are ordered randomly, the probability that we get $a_{1}, \ldots, a_{k}$ in order is

$$
\left(\begin{array}{c}
k \\
m_{1} \cdots m_{k}
\end{array}\right)^{-1}
$$

The result follows from multiplying the previous two displays.

The combined message of the previous two lemmas is that we may assume $\pi$ has between $\delta \log n$ and $10 \log n$ cycles (for any slowly decaying $\delta$ ), while, conditional on $k$, these cycles are distributed roughly independently according to a harmonic weighting.

For any set $S$ of integers, let us call $h_{S}=\sum_{j \in S} 1 / j$ the harmonic weight of $S$. If $P$ is any set of prime numbers, a positive integer $n$ is $P$-smooth iff all prime divisors of $n$ are elements of $P$.

Lemma 5. Let $N \geqslant 1$. Let $P$ be the set of all primes $p \leqslant N$, as well as some o $(N)$ further primes. Then the harmonic weight of the set of $P$-smooth numbers is

$$
(1+o(1)) e^{\gamma} \log N
$$

where $\gamma$ is the Euler-Mascheroni constant.

Proof. The harmonic weight of the set of $P$-smooth numbers is

$$
\prod_{p \in P}(1-1 / p)^{-1}
$$

By Mertens' third theorem we have

$$
\prod_{p \leqslant N}(1-1 / p)^{-1} \sim e^{\gamma} \log N
$$


On the other hand we have

$$
\prod_{p \in P, p>N}(1-1 / p)^{-1}=\exp \sum_{p \in P, p>N} O(1 / p)=e^{o(1)} .
$$

Recall that $\delta=1 / \log \log \log n$, and $\eta=e^{-10 / \delta}=\frac{1}{(\log \log n)^{10}}$. With this choice of $\delta$ and $\eta$ we have the following proposition.

Proposition 6. Let $\pi$ be drawn from $S_{n}$ uniformly at random. Then apart from an event of probability $n^{-1+o(1)}, \pi$ has at least $\delta \log n$ cycles and $\operatorname{ord} \pi$ is divisible by at least $\delta \log n$ primes $p>n^{\eta}$.

Proof. Let $A_{1}, \ldots, A_{Z}$ be the cycle lengths of $\pi$ in a random order. By Lemma 4, provided that $a_{1}+\cdots+a_{k}=n$ we have

$$
\mathbf{P}\left(Z=k, A_{1}=a_{1}, \ldots, A_{k}=a_{k}\right)=\frac{1}{k !} \frac{1}{a_{1} \cdots a_{k}} .
$$

Define sets of primes $P_{i}$ as follows:

(1) Let $P_{0}$ be the set of all primes $p \leqslant n^{\eta}$.

(2) For $0<i \leqslant k$, if $A_{i}$ is $P_{i-1}$-smooth, put $P_{i}=P_{i-1}$. Otherwise pick a prime $p_{i} \notin P_{i-1}$ dividing $A_{i}$ (the smallest such, say), and let $P_{i}=P_{i-1} \cup\left\{p_{i}\right\}$.

Each set $P_{i}$ contains at most $k$ primes $p>n^{\eta}$, so as long as $k=o\left(n^{\eta}\right)$ Lemma 5 implies that the set of $P_{i}$-smooth numbers has harmonic weight at most $2 \eta h_{n}$.

Let $I$ be the set of indices $i \in\{1, \ldots, k\}$ such that $A_{i}$ is $P_{i-1}$-smooth (and hence $P_{i}=P_{i-1}$ ). Assuming $k \geqslant 2 \delta \log n$, if $|I| \leqslant k / 2$ then we find that $P_{k}$ contains at least $\delta \log n$ distinct primes $p>n^{\eta}$, as desired. We will bound the probability that $|I|>k / 2$.

Let $E_{k}$ be the event that $\pi$ has $k$ cycles and $|I|>k / 2$. Then, assuming $2 \delta \log n \leqslant k \leqslant 10 \log n$,

$$
\begin{aligned}
\mathbf{P}\left(E_{k}\right) & =\sum_{I_{0}:\left|I_{0}\right|>k / 2} \mathbf{P}\left(Z(\pi)=k \text { and } I=I_{0}\right) \\
& =\sum_{I_{0}:\left|I_{0}\right|>k / 2} \sum_{\substack{a_{1}, \ldots, a_{k} \geqslant 1 \\
a_{1}+\cdots+a_{k}=n}} \frac{1}{k !} \frac{1_{a_{i} \text { is } P_{i-1} \text {-smooth for each } i \in I_{0}}}{a_{1} \cdots a_{k}} \\
& \leqslant \sum_{I_{0}:\left|I_{0}\right|>k / 2} \frac{1}{k !} h_{n}^{k-\left|I_{0}\right|}\left(2 \eta h_{n}\right)^{\left|I_{0}\right|} \\
& \leqslant \frac{h_{n}^{k}}{k !} 2^{k}(2 \eta)^{k / 2} \\
& \leqslant \frac{h_{n}^{k}}{k !}(8 \eta)^{\delta \log n} \\
& \leqslant \frac{h_{n}^{k}}{k !} n^{-10+o(1)} .
\end{aligned}
$$


Hence

$$
\mathbf{P}\left(\bigcup_{2 \delta \log n \leqslant k \leqslant 10 \log n} E_{k}\right) \leqslant e^{h_{n}} n^{-10+o(1)}=n^{-9+o(1)} .
$$

On the other hand, by Corollary 3 the probability that $\pi$ has either fewer than $2 \delta \log n$ cycles or more than $10 \log n$ cycles is bounded by $n^{-1+o(1)}$. This proves the lemma.

This finishes the proof of part (2) of Theorem 3 .

\section{Proof of Theorem [3, part (1)}

Recall that $\delta=1 / \log \log \log n$, and $\eta=e^{-10 / \delta}=\frac{1}{(\log \log n)^{10}}$.

Lemma 7. Let $m$ be an integer having at least $\delta \log n$ prime divisors $p>n^{\eta}$. Then

$$
\mathbf{P}(\operatorname{ord} \pi=m) \leqslant e^{-c \delta \eta \log ^{2} n} .
$$

Proof. Recall that the cycle lengths of a random permutation can be sampled using the following process. Start by picking $a_{1}$ uniformly from $\{1, \ldots, n\}$. If $a_{1}<n$, pick $a_{2}$ uniformly from $\left\{1, \ldots, n-a_{1}\right\}$, etc. The process continues until $a_{1}+\cdots+a_{k}=n$.

Fix a set $P$ of $\lceil\delta \log n\rceil$ prime divisors $p>n^{\eta}$ of $m$. Now sample $\pi \in S_{n}$ using the process just described. For each fixed $i$ and $p$, the probability that $a_{i}$ is divisible by $p$ is at most $1 / p$, independently of the previous steps in the process. In fact, for any set of primes $p_{1}, \ldots, p_{t} \in P$, the probability that $a_{i}$ is divisible by each of $p_{1}, \ldots, p_{t}$ is at most $1 /\left(p_{1} \cdots p_{t}\right)$. On the other hand, in order that ord $\pi=m$, for each $p \in P$ there must be an index $i$ such that $a_{i}$ is divisible by $P$.

The event that $\pi$ has more than $(\log n)^{3}$ cycles is negligible (it has probability $\left.o\left(e^{-c(\log n)^{3}}\right)\right)$. On the other hand, the probability that $\pi$ has at most $(\log n)^{3}$ cycles and that for each $p \in P$ there is some $i$ such that $a_{i}$ is divisible by $p$ is bounded by

$$
\begin{aligned}
\left((\log n)^{3}\right)^{|P|} \cdot \prod_{p \in P} 1 / p & \leqslant(\log n)^{O(\log n)}\left(n^{-\eta}\right)^{\delta \log n} \\
& \leqslant e^{-c \delta \eta \log ^{2} n} .
\end{aligned}
$$

This finishes the proof of Theorem 3 .

\section{Conclusion}

While we have established that $\mathbf{P}\left(\right.$ ord $\pi=$ ord $\left.\pi^{\prime}\right)$ is generically larger than $O\left(1 / n^{2}\right)$ but no larger than $n^{-2+o(1)}$, its exact order of magnitude remains mysterious and appears to be linked with arithmetical properties of $n$, as in the proof of Theorem 1, Establishing more precise estimates should be of interest to anyone who considers themself to be a problem solver (in the sense of Gowers's essay [Gow00]), just because it is an easily stated problem 
that is not readily solved. We list here a few related observations and open questions.

(1) What is the liminf of $n^{2} \mathbf{P}$ (ord $\pi=$ ord $\left.\pi^{\prime}\right)$ as $n$ tends to infinity? The integers $n$ constructed by Theorem 1 have a particular arithmetic form. What is the behaviour for $n$ of the form $k !+1$ ?

(2) What is $\max _{m} \mathbf{P}(\operatorname{ord} \pi=m)$, and for what value(s) of $m$ is it attained? Theorem 3 gives an upper bound of $n^{-1+o(1)}$ for this probability. Clearly the max is at least $1 / n$, since $\pi$ is an $n$-cycle with probability $1 / n$. In fact the max is at least $1 /(n-1)$, for the same reason but with $(n-1)$-cycles. The answer may be close to this, but we saw in the proof of Theorem 1 that

$$
\mathbf{P}(\text { ord } \pi=n-k) \geq 1 /(n-k)
$$

for any $k \in K_{n}$, so the maximum can be larger. This problem was mentioned by Erdős and Turán in ET68.

(3) Let $\pi_{n}$ be a random element of $S_{n}$. The quantity $\mathbf{P}\left(\operatorname{ord} \pi_{n}=m\right)$ as a function of $m$ and $n$ can be very sensitive to the value of $n$. For example, if $n$ is prime then $\mathbf{P}\left(\operatorname{ord} \pi_{n}=n\right)=1 / n$ but $\mathbf{P}\left(\operatorname{ord} \pi_{n-1}=\right.$ $n)=0$.

(4) As a generalization of Godin's problem, one might consider symmetric groups of different sizes. Consider random permutations $\left(\pi_{1}, \pi_{2}\right) \in S_{n_{1}} \times S_{n_{2}}$, and estimate the probability they have the same order. An upper bound is immediate from Corollary 3 and the Cauchy-Schwarz inequality:

$$
\begin{aligned}
\mathbf{P}\left(\operatorname{ord} \pi_{1}=\operatorname{ord} \pi_{2}\right) & =\sum_{m} \mathbf{P}\left(\operatorname{ord} \pi_{1}=m\right) \mathbf{P}\left(\operatorname{ord} \pi_{2}=m\right) \\
& \leqslant \mathbf{P}\left(\operatorname{ord} \pi_{1}=\operatorname{ord} \pi_{1}^{\prime}\right)^{1 / 2} \mathbf{P}\left(\operatorname{ord} \pi_{2}=\operatorname{ord} \pi_{2}^{\prime}\right)^{1 / 2} \\
& \leqslant n_{1}^{-1+o(1)} n_{2}^{-1+o(1)} .
\end{aligned}
$$

\section{REFERENCES}

[ABT03] Richard Arratia, A. D. Barbour, and Simon Tavaré. Logarithmic combinatorial structures: a probabilistic approach. EMS Monographs in Mathematics. European Mathematical Society (EMS), Zürich, 2003.

[Dev88] Luc Devroye. Applications of the theory of records in the study of random trees. Acta Inform., 26(1-2):123-130, 1988.

[ET67] P. Erdős and P. Turán. On some problems of a statistical group-theory. III. Acta Math. Acad. Sci. Hungar., 18:309-320, 1967.

[ET68] P. Erdős and P. Turán. On some problems of a statistical group-theory. IV. Acta Math. Acad. Sci. Hungar., 19:413-435, 1968.

$\left[\mathrm{FFG}^{+}\right.$06] Philippe Flajolet, Eric Fusy, Xavier Gourdon, Daniel Panario, and Nicolas Pouyanne. A hybrid of Darboux's method and singularity analysis in combinatorial asymptotics. Electron. J. Combin., 13(1):Research Paper 103, 35, 2006.

[For] Kevin Ford. Anatomy of integers and random permutations course lecture notes.https://faculty.math.illinois.edu/ ford/Anatomy_lectnotes.pdf. 
[God17] Thibault Godin. An analogue to Dixon's theorem for automaton groups. In 2017 Proceedings of the Fourteenth Workshop on Analytic Algorithmics and Combinatorics (ANALCO), pages 164-173. SIAM, Philadelphia, PA, 2017.

[Gow00] W. T. Gowers. The two cultures of mathematics. In Mathematics: frontiers and perspectives, pages 65-78. Amer. Math. Soc., Providence, RI, 2000.

[Gra] Andrew Granville. The anatomy of integers and permutations. https://www.dms . umontreal.ca/ andrew/MSI/AnatomyForTheBook.pdf.

[HT88] Richard R. Hall and Gérald Tenenbaum. Divisors, volume 90 of Cambridge Tracts in Mathematics. Cambridge University Press, Cambridge, 1988.

[NP07] Alice C. Niemeyer and Cheryl E. Praeger. On permutations of order dividing a given integer. J. Algebraic Combin., 26(1):125-142, 2007.

[NPS13] Alice C. Niemeyer, Cheryl E. Praeger, and Ákos Seress. Estimation problems and randomised group algorithms. In Probabilistic group theory, combinatorics, and computing, volume 2070 of Lecture Notes in Math., pages 35-82. Springer, London, 2013.

[PW13] Robin Pemantle and Mark C. Wilson. Analytic combinatorics in several variables, volume 140 of Cambridge Studies in Advanced Mathematics. Cambridge University Press, Cambridge, 2013.

[Rai11] Alexander Raichev. New software for computing asymptotics of multivariate generating functions. ACM Commun. Comput. Algebra, 45(3-4):183-185, 2011.

[Thi16] Thibo. What is the probability that two random permutations have the same order? MathOverflow, 2016. http://mathoverflow.net/a/230276 (visited on 2018-June-02).

[Tho20] James Thomas. Three Problems in the Asymptotic Order of Group Elements. PhD thesis, Drexel University, 2020.

[War78] Richard Warlimont. Über die Anzahl der Lösungen von $x^{n}=1$ in der symmetrischen Gruppe $S_{n}$. Arch. Math. (Basel), 30(6):591-594, 1978.

[Wil86] Herbert S. Wilf. The asymptotics of $e^{P(z)}$ and the number of elements of each order in $S_{n}$. Bull. Amer. Math. Soc. (N.S.), 15(2):228-232, 1986.

Department of Mathematics, Drexel University, Philadelphia, Pa, 19104

Email address: ha627@drexel.edu

Mathematics Department, Xavier University of Louisiana

Email address: cburnet2@xula.edu

Department of Pure Mathematics and Statistics, University of Cambridge,London

Email address: eberhard.math@gmail.com

Department of Mathematics, Drexel University, Philadelphia, Pa, 19104

Email address: eschmutz@math.drexel.edu

Department of Mathematics, Drexel University, Philadelphia, Pa, 19104

Email address: jjt94@dragons.drexel.edu 